\title{
Alcances y límites del multiculturalismo liberal desde un enfoque de género interseccional
}

\author{
Scope and limits of liberal multiculturalism \\ from an intersectional gender approach
}

\author{
Sabina García-Peter / sabina.garcia.peter@fu-berlin.de \\ Margherita-von-Brentano-Zentrum, Freie Universität Berlin, Alemania \\ Luis Villavicencio-Miranda / luis.villavicencio@uv.cl \\ Universidad de Valparaiso, Chile
}

\begin{abstract}
The article presents a critical analysis of two of the most influential theoretical positions within liberal multiculturalism -represented by Will Kymlicka and Charles Taylor-. Using an intersectional theoretical and methodological approach, the scope and limits from both positions are shown. The analysis shows that cultural diversity is an issue that acquires new and rich hues when approached holistically remarking the intersections of gender inequalities with other mechanisms of social inequality, such as 'race'/ethnicity and social class. It concludes that only by integrating and intersecting the study of these variables in the study of cultural diversity will it become possible to advocate for democratic politics that emphasizes the specific nature of the differences, but within the principles of equality and justice.
\end{abstract}

Key words: cultural diversity, liberal multiculturalism, intersectionality, gender, inequality.

Resumen: El artículo presenta un análisis crítico de dos de las posturas teóricas de mayor influencia dentro del multiculturalismo liberal -representadas por Will Kymlicka y Charles Taylor-. Con base en una perspectiva teórico-metodológica de género interseccional, se muestran los alcances y límites de ambas posturas. El análisis señala que el estudio de la diversidad cultural es un asunto que adquiere nuevos y ricos matices cuando se aborda holísticamente, colocando en el centro la relación de las desigualdades de género con otros mecanismos de desigualdad social, como la "raza"/etnicidad y la clase social. Para concluir se propone que sólo al integrar y conectar el estudio de estas variables en la investigación sobre la diversidad cultural, es posible abogar por una política democrática que ponga énfasis en la naturaleza específica de las diferencias, pero dentro los principios de la igualdad y la justicia.

Palabras clave: diversidad cultural, multiculturalismo liberal, interseccionalidad, género, desigualdad. 


\section{Introducción $^{1}$}

El multiculturalismo liberal ${ }^{2}$ (Kymlicka, 2003: 59-63) es considerado una de las respuestas más influyentes respecto a cómo estudiar la diversidad cultural. ${ }^{3}$ Surge a partir de la segunda mitad del siglo XX, principalmente en países del Norte, como Canadá y Estados Unidos, para hacer referencia a una serie de demandas por parte de grupos minoritarios - gays, afroamericanos, feministas, grupos étnicos, entre otros- que van desde lo jurídico hasta lo educativo.

Como respuesta teórico-académica a la lucha social por el reconocimiento, ${ }^{4}$ el debate multiculturalista ha generado una confusión respecto a la red de conceptos que lo conforman y una polémica sobre la definición de los términos al que se refiere. Los marcos conceptuales desarrollados por diferentes autores y autoras desde distintas corrientes definirán, por ejemplo, en qué medida se va a privilegiar cierto orden propio e inmodificado de cada una de las diferentes culturas.

Términos clave aquí son el de "diferencia” y "cultura": cómo se les entiende, delimita y refuerza semejanzas o divergencias, rasgos o supuestos, aportando un grado siempre interesante, nunca neutro, y en la mayor parte de las veces inestable, de construcción y comprensión ad hoc. En este sentido, si bien los límites y alcances de las concepciones sobre la diversidad cultural han sido 1 El presente trabajo forma parte del Proyecto FONDECYT (Chile) núm. 1120566, titulado "Ciudadanía para las mujeres en una sociedad multicultural. Hacia la construcción de una concepción deliberativa con vocación universal y su impacto institucional".

2 En su obra más reciente Kymlicka (2001: 39-42) ha dejado de utilizar la expresión culturalismo liberal (liberal culturalism) que ocupó hasta su libro La politica vernácula y ha preferido optar unívocamente por la expresión multiculturalismo liberal (liberal multiculturalism) (Kymlicka, 2007: 61-86). Si bien este cambio no es decisivo, nos parece adecuado pues se conecta con la distinción analíticamente útil -para efectos de ordenar el debate- entre la voz "multicultural" y la expresión "multiculturalismo". "El término 'multicultural' se refiere al hecho de la diversidad cultural, la expresión 'multiculturalismo' a una respuesta normativa para ese hecho" (Parekh, 2006: 6). En el ámbito hispanoamericano, De Lucas (2001: 62-64) ha hecho suya la misma diferenciación.

3 El hecho de la diversidad cultural copa hoy buena parte de la agenda política y teórica (Parekh, 1999; Kymlicka, 2002: 327-328; Torbisco, 2006: 1-9). Para un análisis general, véase Pérez de la Fuente (2005: 254), Villavicencio (2010a y 2010b). Como no hay una sola clase de multiculturalismo (Cfr. Parekh, 2002: 133-150), es más adecuado hablar de multiculturalismos (De Lucas, 2001: 61-102; Banting y Kymlicka, 2006: 9; Young, 2000: 31-69). 4 La lucha por el reconocimiento es, en buena medida, una crítica a la concepción liberal de ciudadanía liberal (Song, 2007: 68; Fraser, 2000; Delanty, 2010: 59; Modood, 2007: 68-70). 
discutidos ampliamente - no sólo desde la mirada del multiculturalismo, sino también del interculturalismo-, estas discusiones han estado enmarcadas dentro del debate en torno al concepto de cultura, como si la "diferencia cultural" fuera la única fuente de diversidad.

En este artículo se pretende ir un paso más allá, enriqueciendo la discusión en torno a las conceptualizaciones de la diversidad cultural desde una perspectiva interseccional, como una manera de ampliar el debate al presentar una forma alternativa de analizar e interpretar este fenómeno.

El artículo se sirve del enfoque de la interseccionalidad para plantear una posición teórico-crítica respecto al panorama de las teorizaciones y conceptualizaciones en relación con la diversidad cultural. Se revisan dos de las posturas teóricas más importantes en torno al multiculturalismo liberal con el objetivo de (re)conceptualizar sus definiciones desde una perspectiva teórico-metodológica de la interseccionalidad. Para ello se toman como base los postulados de Will Kymlicka (1989, 1995) y Charles Taylor (1992), cuyas contribuciones dentro del ámbito de la "primera ola de multiculturalismo liberal" -que incluye los trabajos de ambos y los de otros autores desde finales de la década de 1980 y principios de 1990- son las más influyentes hasta hoy en día a nivel global (Kymlicka, 2007).

\section{Una alternativa a las políticas de la identidad: el enfoque de la interseccionalidad}

El enfoque de la interseccionalidad ${ }^{5}$ ha sido considerado la contribución teórica más importante que los estudios sobre las mujeres han hecho hasta ahora (McCall, 2005; Davis, 2008; Nash, 2008; Guzmán-Ordaz, 2009). Dicho enfoque ha venido adquiriendo un protagonismo decisivo para enfrentar la comprensión y resolución teórico-metodológica del estudio de las desigualdades sociales al tomar en cuenta, simultánea y relacionalmente, la complejidad derivada de las múltiples experiencias de las personas (GuzmánOrdaz, 2011:2).

$5 \mathrm{Al}$ igual que el concepto de multiculturalismo, la interseccionalidad ha generado una extensa discusión en términos de su significado: mientras algunos la distinguen como un concepto (Crenshaw, 1994), otros se refieren a ésta como una perspectiva (Shield, 2008) o un paradigma (Hancock, 2007). Ahora bien, es en torno a su propia ambigüedad e inconclusión epistemológica que adquiere su fortaleza teórico-metodológica al encontrarse en constante revisión y expansión tanto disciplinaria como argumental y analítica (GuzmánOrdaz, 2011). 
El debate teórico en torno a la interseccionalidad adquiere relevancia, sobre todo, dentro del contexto de lo que se conoce como "feminismos de la tercera ola" -identificados como tal a partir de la década de 1990-, cuya preocupación se sitúa, principalmente, en dar cuenta de la importancia que revisten las diferencias.

Estos movimientos surgieron como una respuesta a las debilidades presentadas por el llamado "feminismo de segunda ola", al plantear la premisa de la existencia de múltiples "modelos" de mujer, los cuales están determinados por cuestiones sociales, étnicas, de nacionalidad y de religión, entre otros. Los debates dentro de esta corriente buscaban alejarse del esencialismo y las definiciones de feminidad asumidas en ocasiones como universales y que sobreestimaban las experiencias de las mujeres occidentales, de clase media y blanca dentro del pensamiento feminista. ${ }^{8}$

El Black Feminism y el Feminismo Chicano son dos propuestas radicales que sirven como antecedente, al posicionarse en contra de los efectos del colonialismo desde una visión materialista, antirracista y antisexista (Curiel, 2008) e introducir críticas a los vacíos y la falta de reconocimiento que los feminismos hegemónicos habían hecho hasta ese momento sobre la condición de las "otras", las diferentes, las marginales; cuestionando principalmente la visión esencialista y estática de la categoría "mujer" (Guzmán-Ordaz, 2009: 4).

Es precisamente a partir de estas experiencias que más tarde aparece el concepto de interseccionalidad (intersectionality), el cual fue acuñado por primera vez en términos legales y académicos por Kimberlé Crenshaw (1994), ya hacia finales de la década de 1980 (Yuval-Davis, 2006; Davis, 2008; Nash, 2008; Winker y Degele, 2009).

6 Aunque es en este contexto donde el término interseccionalidad como tal adquiere fuerza, fueron las feministas racializadas, afrodescendientes e indígenas las primeras en profundizar, ya desde la década de 1970, en la imbricación de diversos sistemas de dominación. El colectivo Río Combahee, constituido por mujeres y lesbianas de color, fue el primero en proponer en 1977 que la raza, sexo, clase, sexualidad debían ser entendidas como co-sustanciales, no separadas unas de otras (Curiel, 2014). Años después las feministas chicanas profundizaron en torno a esto (Cfr. Moraga y Anzaldúa, 1981).

7 El surgimiento del feminismo de la segunda ola se asocia a la nueva izquierda antiimperialista y a un cuestionamiento del paternalismo del Estado de Bienestar socialdemócrata y la familia burguesa, así como la denuncia del androcentrismo que subyacía al capitalismo (RigatPflaum, 2014).

8 Un análisis general sobre la teoría feminista considerada como una de las teorías críticas del liberalismo, puede verse en Squella et al. (2012: 221-271). 
La interseccionalidad puede ser entendida como un enfoque crítico que permite comprender las posiciones de las personas (identidad) en contextos diversos y a partir de las conexiones entre las estructuras de género, "raza"/ etnia y clase social, y la manera como la intersección de éstas y otras estructuras sociales, o estatus epistémicos de la diferencia, pueden producir contextos complejos de desigualdades que pueden, a su vez, ser analizados en términos de poder y a través de distintos niveles (Anthias y Yuval-Davis, 1992; Anthias, 1998, 2008; Andersen, 2005; McCall, 2005; Winker y Degele, 2009).

Para Anzaldúa (1987), la "raza” y la clase social contribuyen - del mismo modo que lo harían el género y la preferencia sexual- en la manera como nos percibimos a nosotros mismos y cómo somos percibidos por los demás, jugando un papel constitutivo en la tarea de emprender la construcción de las identidades. De la lectura de Anzaldúa se desprende una crítica a las condiciones opresivas (para las mujeres) inherentes a los distintos sistemas culturales dominados por una supremacía de lo masculino. Estas condiciones son difíciles de separar en la medida que se constituyen como experiencias simultáneas de opresión: no podemos criticar el racismo, sin hacer referencia al sexismo y al clasismo, y viceversa.

En este sentido, considerar la intersección del género con otras categorías como la "raza"/etnia o la clase social en el análisis y comprensión de la diversidad cultural resulta relevante en la medida que el género debe ser entendido no sólo como el conjunto de creencias, prescripciones y atribuciones que se construyen socialmente en relación con lo "femenino", sino también en relación con lo "masculino". "Esto es clave en la medida que las culturas son definidas a partir de un concepto de sujeto masculino hegemónico, aun cuando muchas veces se pretende que éstas no tienen género.

La interseccionalidad permite no sólo dar cuenta del olvido de las mujeres y reconocer la diversidad sexual, sino también criticar las masculinidades hegemónicas que se (re)producen y ocultan en lo "universal", así como dar cuenta de éstas como construcciones sociales. La crítica hacia el esencialismo cultural no es sólo porque éste estereotipifica lo femenino, sino también lo masculino, a la vez que suele referir sólo a las minorías dejando de lado las características y diferencias existentes dentro de las mayorías.

Se entiende entonces la interseccionalidad como una alternativa a la política de la identidad que permite considerar las diferencias intragrupales, subvirtiendo las separaciones entre género, "raza"/etnia y clase social como elementos separados y disyuntivos. En este sentido, al hablar de diversidad cultural queremos hacer hincapié en la necesidad de entenderla a partir de su carácter inherente y constitutivamente interseccional.

9 Sobre la idea de género, véase Benería (1987: 46) y Sánchez (2002: 359). 
Como tal, la diversidad cultural constituye un fenómeno intrínsecamente formado por distintas condiciones que la modelan conjuntamente de una manera que no puede ser definida a priori, poniendo de relieve su origen complejo y su interconexión originaria con diferentes condiciones de identificación y discriminación social como el género, la "raza"/etnia y la clase social, entre otras. Esta postura se sustenta en el principio de que ninguna cultura debería ser reivindicada sin haber hecho conciencia de aquellos aspectos sexistas, racistas y clasistas que la constituyen.

\section{Alcances y límites del multiculturalismo liberal}

El enfoque de la interseccionalidad puede ser utilizado como una herramienta teórica-metodológica para reflexionar sobre los límites y alcances de los postulados de dos de los autores más representativos dentro del multiculturalismo liberal. El objetivo de este ejercicio es revisar las posibilidades de conceptualizar la diversidad cultural desde una perspectiva que incluya en su definición no sólo la importancia de las diferencias culturales, sino también su relación e interacción con otras categorías de poder, así como las desigualdades derivadas de éstas.

\section{Will Kymlicka y la Ciudadanía Multicultural}

Dentro del debate en torno a la pluralidad cultural podemos ubicar a Kymlicka como uno de los mayores exponentes del multiculturalismo liberal. Kymlicka (1996: 13-19; 2003: 29-42; 2007: 61), al igual que otros multiculturalistas, como Parekh (2006: 179-185; 2008: 80-98), parten de un punto de vista común: su oposición al modo en que el liberalismo aborda la diversidad cultural y el diseño institucional que va de la mano, esto es, la construcción de un Estado-nación unitario y homogéneo.

A pesar de ese diagnóstico compartido, Kymlicka difiere de los multiculturalistas respecto del modo en que habrá de enfrentarse al problema de las reivindicaciones multiculturales, algo que no debería sorprendernos pues, después de todo, nuestro autor es liberal. ${ }^{10}$ Revisemos brevemente los planteamientos de Kymlicka.

10 Desde la perspectiva de Parekh (2006: 195), a diferencia de Kymlicka, nada se resta del debate, es decir, no existen derechos previos innegociables, sólo una apuesta -algo incierta e ingenua, diría un liberal- por un diálogo intercultural. Para una comparación detallada entre estos dos autores véase Villavicencio (2012). 
Su tesis pone el acento en el rol que han jugado y juegan las fronteras territoriales en relación con el goce de los derechos y la pretensión de justicia, así como los retos que esto implica para las democracias liberales de posguerra, las cuales se han visto sometidas a un intensivo proceso de diversificación y fragmentación cultural que va en aumento (Pérez, 2007). Este fenómeno lleva a Kymlicka (1996: 14) a plantear la intención de "identificar algunos conceptos y principios claves a tomar en consideración y, finalmente, clarificar los fundamentos básicos de un enfoque liberal del problema de los derechos de las minorías".

El filósofo canadiense plantea que el multiculturalismo liberal pretende realzar la importancia de la identidad cultural protegiendo a las minorías nacionales y étnicas, pero garantizando la vigencia de ciertos principios liberales básicos (Kymlicka, 2003: 59). El Estado debe adoptar, entonces, políticas públicas que aseguren a los miembros de los diversos grupos étnicos y nacionales expresar y promover sus propias identidades, pero rechazando aquellas culturas que busquen la imposición de las mismas.

El punto de partida de esta posición es comprender la relevancia del contexto social como requisito para que exista una elección autónoma auténtica y significativa. Como sostiene Raz (2001:192), "sólo a través de la socialización en una cultura podemos canalizar las opciones que dan sentido a la vida". La conexión del planteamiento con la tesis comunitarista que defiende la naturaleza contextual de los seres humanos es evidente, pero con un muy preponderante matiz: esa naturaleza sólo tiene valor en cuanto contribuya a asegurar para cada persona un marco de opciones cualitativamente significativas. ${ }^{11}$

Así, las culturas ya no son relegadas a la esfera privada, como plantea el liberalismo, sino que juegan un papel protagónico en la conformación de aspectos decisivos en la construcción de la identidad, como es el caso de la lengua, la educación o ciertos símbolos nacionales (Kymlicka, 1996: 152164) que se traducen en medidas que "van desde las políticas educativas multiculturales a los derechos lingüísticos, pasando por las garantías de representación política y por la protección constitucional de los tratados con los pueblos indígenas" (Kymlicka, 2003: 63).

Los Estados modernos están constituidos -casi siempre- por diversos grupos étnicos y/o nacionales, lo cual demanda superar el discurso liberal de la neutralidad y aceptar que al interior de ellos habrán de tomarse inevitables decisiones culturales que afectan a esos grupos. Surge así la necesidad de reconocer algunos derechos de grupo, bajo el alero de una ciudadanía

11 Una aproximación crítica y sistemática al debate liberal-comunitarista puede verse en Villavicencio (2014). 
diferenciada y orientada culturalmente, para dotar a las minorías de los mecanismos necesarios para enfrentar las discriminaciones que pueden sufrir de parte de la cultura mayoritaria.

Para el multiculturalismo liberal, la dicotomía entre derechos colectivos y derechos individuales es falsa, pues deben distinguirse con cuidado dos tipos de restricciones o protecciones asociadas a ellos: por una parte, se encuentran las protecciones externas, esto es, aquellas que autorizan al grupo -étnico o nacional- a ser tratado con igual consideración y respeto que otros grupos equivalentes; ${ }^{12}$ y, por otra, están las restricciones internas, o sea aquellas que impiden al grupo coaccionar a sus miembros, impidiéndoles revisar su concepción del bien. ${ }^{13}$

Los derechos colectivos entendidos como protecciones externas son absolutamente compatibles con una teoría liberal de los derechos que pretenda fomentar la autonomía; mientras, las restricciones internas son inaceptables (Kymlicka, 1996: 57-76).

De esta forma, al concebir los derechos de las minorías como un problema, Kymlicka (2003: 59) plantea una propuesta para enfrentar el reto del multiculturalismo de un modo liberal: se debe proteger a las minorías mediante derechos específicos para los grupos. Esta propuesta política significa colocar, junto a los derechos individuales del liberalismo y la democracia, derechos diferenciados en función de la pertenencia a un grupo.

Por tanto, Kymlicka (1996: 13-19; 2003: 29-42; 2007: 61) se propone reformar o ampliar la teoría liberal de los derechos individuales, mostrando que ésta es compatible con la existencia de derechos para grupos (Pérez, 2007). El desafío del multiculturalismo radica en cómo evitar que las sociedades mayoritarias impongan sus instituciones a quienes pertenecen a una cultura diferente.

Kymlicka (2003: 82-87) es claro en señalar y criticar que el concepto de multiculturalismo suele utilizarse de una forma muy amplia para englobar una extensa gama de grupos sociales no étnicos que, por diversas razones, han sido excluidos o marginados del núcleo mayoritario de la sociedad -por ejemplo, los discapacitados, los gays y las lesbianas, las mujeres, etcétera-. Para Kymlicka (1996: 36), "un Estado es multicultural si sus miembros pertenecen a naciones diferentes (un Estado multinacional) o si bien éstos han emigrado de diferentes naciones (un Estado poliétnico), siempre y cuando ello suponga un aspecto importante de la identidad personal y la vida política”.

12 Por ejemplo, los derechos a una lengua propia, el derecho a un estatuto especial de posesión de tierras o el derecho al gobierno, total o parcialmente autónomo.

13 Típicamente el derecho a sancionar a los individuos que se aparten de las creencias colectivas. 
Desde el enfoque de la interseccionalidad, la visión de Kymlicka puede resultar parcial en relación con el sentido del desafío multicultural. Al respecto es posible mencionar al menos tres críticas a los postulados de este autor que podrían ampliarse desde dicho enfoque.

Primero, la teoría de Kymlicka no permite dar cuenta de los procesos de minorización de los grupos dentro de las sociedades -los cuales "se presentan como ahistóricos, como si las minorías siempre hubieran estado ahí y no fueran cambiantes y parte de un proceso histórico más amplio y complejo" (Dávila Figueroa, 2013: 134)- donde convergen relaciones sociales que son transversales y simultáneas a la vez.

Junto con ello, Kymlicka pierde de vista a los grupos mayoritarios y dominantes, poniendo un excesivo énfasis en las minorías, sin considerar que estas últimas sólo "existen" en relación con una mayoría que también es internamente diversa. En este sentido, el concepto de cultura propuesto por Kymlicka (2003: 78) es estrecho en la medida que no considera las "constantes creaciones, recreaciones y negociaciones de fronteras imaginarias entre 'nosotros' y el/los 'otro(s)"' (Benhabib, 2006: 33). El "nosotros" siempre supone un "otro" del que se diferencia y a partir del cual se define, y ese "otro" debe ser comprendido a partir del entrecruzamiento de diferentes ejes de diferenciación.

Segundo, y siguiendo la crítica feminista al multiculturalismo, el énfasis en las culturas minoritarias no toma, además, en consideración que dentro de estos grupos es posible identificar otras minorías que también requieren atención, como las mujeres en el contexto de las culturas patriarcales.

La crítica de Susan Moller Okin (1999) ha sido clave en develar la tensión entre multiculturalismo y feminismo, la cual se basa en la existencia de un conflicto entre los reclamos de las culturas o religiones minoritarias y la norma genérica de igualdad aceptada en todos los Estados liberales, señalando que el multiculturalismo es de por sí malo para las mujeres.

En esta dirección, los argumentos de Kymlicka (1990: 239-62 en Moller Okin, 1999) a favor del multiculturalismo no toman en cuenta lo que él mismo reconoce en otros escritos: que la subordinación de las mujeres es a menudo informal y privada, y que virtualmente ninguna cultura en el mundo, minoritaria y mayoritaria, puede pasar el test que él propone de "ninguna discriminación" en la esfera privada.

Sin embargo, la crítica feminista de Moller-Okin sólo puede ser asumida como un guiño a un análisis que considere un enfoque interseccional, ya que desde este último se evita esencializar la cultura ${ }^{14}$ al dar cuenta de que las

14 Seyla Benhabib (2006) ve en la argumentación de Moller-Okin (1999) un problema en la medida que ésta descansa en una comprensión monolítica de las culturas. Eso le impide 
diferencias internas son un producto de la simultaneidad y cruce de diversos ejes de diferenciación.

En este sentido, si bien Moller-Okin (1999) puntualiza en la importancia de considerar a las mujeres como grupo minoritario dentro de las culturas patriarcales, cuyos derechos, en el marco del liberalismo, están por sobre los derechos culturales, existe el riesgo de "revivir las dinámicas coloniales de la 'otredad'” (Deckha, 2004: 22). La interseccionalidad se presenta así como un modelo de análisis sistemático que hace más sencillo de-construir y analizar las dinámicas internas de las agrupaciones de identidades colectivas desde una perspectiva no colonialista.

Tercero, la visión de Kymlicka centrada en los derechos de las minorías no da cuenta de las relaciones de dominación y subordinación que deben ser vistas desde una perspectiva que tome en consideración las desigualdades de género, clase y "raza”/etnia.

En la medida que el autor presume que el otorgamiento de reconocimiento y acomodo a la diferencia cultural implica una desaparición de los problemas de orden estructural, su postura -marcadamente liberal- pierde de vista la importancia de la redistribución que, junto al reconocimiento, pretende lograr la justicia social, más allá de la integración, asumiendo que hay problemas de orden político y económico, aparte del cultural.

Fraser (2000) critica al multiculturalismo $-\mathrm{y}$ al antiesencialismo ${ }^{15}-\mathrm{de}$ no ser capaz de relacionar una política cultural de identidad y diferencia con una política social de justicia e igualdad al basarse en un enfoque unilateral de la identidad y la diferencia.

Esta tendencia a tratar la diferencia como si fuera de naturaleza exclusivamente cultural, debe ampliarse mediante un enfoque que explique que las cuestiones relativas a ésta no pueden ser analizadas independientemente de la desigualdad material, de las diferencias de poder entre los grupos y de las relaciones de dominación y subordinación que se dan dentro del sistema.

aprehender el juego de las complicidades, lealtades y tensiones que se reproducen sin cesar al interior de ellas. Es decir, se pierde la dialéctica de los derechos políticos y de las identidades culturales, y traslada al multiculturalismo un relativismo cultural y moral que desvía el eje fundamental del debate (Femenías, 2008: 180).

15 Fraser (2000) señala que en la actualidad los debates en torno a las identidades de grupo y la diferencia cultural se dividen en dos corrientes relacionadas: el antiesencialismo, que reconceptualiza la identidad y la diferencia como construcciones discursivas creadas performativamente a través de los procesos culturales que los elaboran y sustentan (Piastro, 2014); y el multiculturalismo, que adopta un punto de vista positivo en relación con las diferencias e identidades de grupo, intentando revalorizarlas y fomentarlas. 
Es posible profundizar en la limitación de la teoría de Kymlicka (1996; 2003) deteniéndonos en las críticas que Parekh (2006: 99-109) ha realizado ${ }^{16}$ a su trabajo. El primer reproche se centra en la concepción más bien estrecha y esencialista con que utiliza el término cultura que impacta directamente en la nítida distinción y en el diverso peso moral que tendrían las demandas de dos tipos de minorías culturales: las nacionales y los inmigrantes voluntarios.

Ese trato diferenciado encuentra su fundamento en las mayores o menores posibilidades que posee cada clase de minoría para proporcionar a sus miembros una cultura societal. Ya que las minorías nacionales - al haber sido subsumidas por un Estado mayor- tenían con anterioridad una cultura societal propia, cumplen con las condiciones mínimas para proporcionar a sus miembros el necesario marco para desarrollarse autónomamente y, por ende, se les debe reconocer el derecho a mantener o emprender su propia construcción nacional y gozar de alguna forma de autogobierno.

"De hecho, su cultura societal les proporciona un contexto más satisfactorio del que habrían tenido en caso de que se les hubiera exigido integrarse en la sociedad principal, ya que es la cultura con las que esas minorías se identifican y están más familiarizadas" (Kymlicka, 2003: 81).

Los inmigrantes legales, continúa Parekh (2006: 99-109), constituyen el otro extremo. No son minorías nacionales sino étnicas, no están territorialmente concentradas ni poseen una institucionalidad completa, su cultura ha sido desarraigada de su contexto original, y no puede reproducirse como tal en otro medio. Además, el hecho de haber emigrado en búsqueda de mejores expectativas también supone que los inspira un genuino deseo de integrarse a la nueva sociedad.

Por estas razones, los inmigrantes no estarían habilitados para ejercitar (y debiesen dejar de demandar) el derecho al autogobierno y a su autonomía cultural, aunque sí pueden exigir mantener algunas de sus prácticas asociadas con su identidad etnocultural, que se traducirían en diversas facultades y exenciones que Kymlicka (2003: 75 y 76) llama derechos de acomodo.

En opinión de Parekh (2006: 103), la cuestión clave es que no existe razón de peso para hacer tal distinción. Muy por el contrario, esa clasificación no hace más que reflejar una larga tendencia histórica liberal de delinear, por un lado, un claro contraste entre los grupos étnicos y las naciones privilegiando a estas últimas; $y$, por otro, establecer una clara diferencia entre inmigrantes y ciudadanos.

16 Hay otro grupo de objeciones intraliberales a la teoría de Kymlicka. La más llamativa es la de Barry (2002: 131-146). Hemos analizado con cierta profundidad esa visión en Villavicencio (2010b). 
Apoyándose en la misma distinción trazada (entre minorías nacionales y étnicas), Carens (2000) y Young (1997) han criticado la visión rígida que despliega Kymlicka (1996; 2003) al ocuparse solamente de los casos antagónicos, omitiendo las situaciones intermedias como sería el caso de grupos oprimidos que, sin pertenecer a aquellas minorías en las cuales se concentra Kymlicka, sí requerirían el reconocimiento de algunos de los derechos de acomodo (Carens, 2000: 52-87; Young, 1997).

Nos parece que sería el caso, sin duda, de las minorías sexuales y de las mujeres (Cfr. Young, 1997: 48-53; 2000: 89-93). No menos relevante es que Kymlicka olvida -como ya hemos dicho-que las minorías se constituyen y comportan, en la realidad, de modo complejo, obviando que las desigualdades derivadas de las diferencias culturales deben ser entendidas como el resultado del entrecruzamiento de los efectos de éstas con otros importantes ejes de diferencia -como el género, la clase, la etnicidad, la raza y la sexualidad-, lo cual dificulta su encasillamiento.

Pensemos, por ejemplo, en los mapuches en Chile, quienes son al mismo tiempo una minoría nacional, étnica y/o social dependiendo, entre otros factores relevantes, de la ubicación geográfica donde se encuentran; a la vez que el género y la clase social constituyen elementos interdependientes respecto a cómo son vivenciadas las desigualdades.

\section{Charles Taylor y la Politica del Reconocimiento}

Otra postura relevante dentro del multiculturalismo liberal es la representada por Charles Taylor, quien problematiza el reconocimiento de la identidad de los grupos minoritarios y el derecho a la diferencia de cada grupo en contextos multiculturales. Si bien Taylor (1993: 31-32) está consciente de la importancia de la universalidad de los derechos y de la igualdad, es preponderante para él atender el reconocimiento de las diferencias sosteniendo que este discurso:

[...] se ha vuelto familiar para nosotros en dos niveles: primero, en la esfera íntima, donde comprendemos que la formación de la identidad y del yo tiene lugar en un diálogo sostenido y en pugna con los otros significantes. Y luego en la esfera pública, donde la política del reconocimiento igualitario ha llegado a desempeñar un papel cada vez mayor. [...] Con la política de la dignidad igualitaria lo que se establece pretende ser universalmente lo mismo, una "canasta" idéntica de derechos e inmunidades; con la política de la diferencia, lo que pedimos que sea reconocido es la identidad única de este individuo o de este grupo, el hecho de que es distinto de todos los demás. 
En este sentido, siguiendo a Taylor, lo que le interesa a los defensores de las políticas de la diferencia es poner el acento en el reconocimiento de la identidad única y original de cada individuo y comunidad, la cual debe ser protegida para evitar que se vea homogeneizada por la identidad de aquellas comunidades hegemónicas.

En la esfera pública, Taylor (1993: 52) plantea el problema de si una sociedad democrática puede conciliar el trato igualitario para todos los individuos con el reconocimiento de las diferencias específicas que dentro de esa misma sociedad se manifiestan o si el liberalismo asume más bien una posición "ciega a la diferencia".

Sin embargo, el autor niega que eso sea así necesariamente y reconoce que dentro del pensamiento liberal, y en algunas sociedades liberales, se manifiesta una posición distinta. A partir de esto plantea la existencia de dos tipos de Estados liberales: el que pretende ser neutral con el argumento de que los derechos individuales no pueden ser restringidos de ninguna manera, garantizando la igualdad de éstos para todos; y el que garantiza el respeto a la diferencia y no pretende ser neutral.

El primero representa la política de la dignidad y consiste básicamente en que los hombres libres e iguales tienen los mismos derechos; por ende, la función del Estado es proteger y asegurar tales derechos; en el segundo cada individuo y cada grupo poseen una identidad y una particularidad que debe ser respetada, por lo cual se exige al Estado la protección de un conjunto de prácticas, tradiciones y valores que harían posible que los individuos de la comunidad política se identificaran con determinado ideal del bien común.

Taylor está más cerca del segundo de estos liberalismos. Sus argumentos tienen que ver, en parte, con la afirmación del principio de respeto a las minorías y con el hecho de que el multiculturalismo es hoy una realidad que se extiende por el mundo y exige una política abierta al reconocimiento de las diferenciaciones culturales y de las metas colectivas.

Una vía para resolver el conflicto entre las interpretaciones que los partidarios de las políticas de la diferencia y los defensores del universalismo hacen del discurso moderno del reconocimiento, concebida por Taylor, es la de un liberalismo comprometido sustancialmente con ciertos fines colectivos, o sea, la de un liberalismo de vocación comunitarista que no busque homogeneizar las identidades culturales. Desarrollaremos esta idea con más detención.

El comunitarismo de Taylor pretende demostrar que las cosas adquieren relevancia y nuestras elecciones cobran sentido sólo al ser contrastadas con unos marcos de inteligibilidad que el autor llama "horizontes de significado común”. Éstos son ineludibles, por lo tanto: 
una de las cosas que no podemos hacer, si tenemos que definirnos significativamente, es suprimir o negar los horizontes contra los que las cosas adquieren significación para nosotros. Éste es el tipo de paso contraproducente que se da con frecuencia en nuestra civilización subjetivista. Al acentuar la legitimidad de la elección entre ciertas opciones, muy a menudo nos encontramos con que privamos a las opciones de su significación (Taylor, 1994: 72).

Para Taylor (1983: 223-224), las democracias modernas se enfrentan a lo que él ha llamado un "dilema hegeliano". Este desafío puede resumirse así: al no haber principios morales universales que la razón pueda descubrir o construir, ¿cómo se arriba a sistemas morales que no desarraiguen a los seres humanos de las comunidades que constituyen sus focos de identidad, en medio de una sociedad moderna que parece haberse habituado, indefectiblemente, al principio de libertad individual?

Desde luego, en opinión de los comunitaristas, el liberalismo no ofrece una buena respuesta precisamente porque sería insensible a los requerimientos que exigen una consideración más detallada de la propia comunidad y las tradiciones donde estamos insertos, con el fin de descubrir cuáles son los valores comunitarios que compartimos.

La principal razón por la cual el liberalismo es incapaz de enfrentarse a ese desafío radica en que es un muy buen ejemplo de la simplificación que afecta a las éticas modernas, las cuales, inspiradas en la idea kantiana de razón práctica, no logran dar cuenta de la profundidad y complejidad de la moralidad humana. Esto se traduce en dos equivocaciones estructurales del liberalismo: en primer lugar, el afán de fundar una moral con prescindencia de una teoría robusta del bien; y, en segundo, la búsqueda de principios con pretensiones universalistas a costa de una abstracción metodológica excesiva.

Para Taylor la única forma de dotar a los valores liberales de una interpretación adecuada -alternativa al liberalismo rawlsiano- pasa primero por un examen minucioso de la sociedad moderna y sus males. Taylor (1994: 37-47) identifica tres formas de malestar que atormentan a la modernidad y entiende por tales "aquellos rasgos de nuestra cultura y nuestra sociedad contemporáneas que la gente experimenta como pérdida o declive, aún a medida que se 'desarrolla' nuestra civilización”.

El primero de ellos es el individualismo: la libertad moderna se alcanzó a costa de escapar de los horizontes morales que poseíamos en el pasado, los cuales, si bien limitaban a los sujetos, también daban sentido al mundo y a los roles que éstos desempeñaban en la sociedad. De este modo, el individualismo tiene un lado oscuro que podría englobarse en la noción de pérdida de sentido. 
El filósofo canadiense considera que la promoción de una sociedad permisiva y el narcisismo son síntomas claros de este aspecto negativo de la individualidad. Pero junto a este primer rasgo nocivo, Taylor agrega una segunda fuente de malestar: la primacía de la razón instrumental.

Al igual que el individualismo, esta característica de la modernidad ha sido liberadora; sin embargo, el peligro radica "en que aquellas cosas que deberían determinarse por medio de otros criterios se decidan en términos de eficiencia o de análisis 'costebeneficio', que los fines independientes que deberían ir guiando nuestras vidas se vean eclipsados por la exigencia de obtener el máximo rendimiento" (Taylor, 1994: 41).

Por último, el tercer rasgo sombrío de la modernidad se desencadena por la combinación de los anteriores y podría ser identificado como una forma de desencantamiento generalizado, que opera tanto en el plano individual como social.

Estas disfunciones afectan profundamente el proceso político y alteran su forma. Por consiguiente, las personas pueden reaccionar a la exclusión

practicando un tipo de política que parece basada en la creencia de que la sociedad está compuesta, en el mejor de los casos, por ciudadanos mutuamente desinteresados y quizás, en su mayoría, incluso malévolos en relación al grupo en cuestión [...] O bien la respuesta puede ser impulsada por una concepción filosófica de exclusión, digamos, una visión marxista de la sociedad burguesa como irrecuperablemente perdida por la lucha de clases o ciertas visiones feministas de la sociedad liberal como irremediablemente viciada por el patriarcado, de modo que una invocación de la comunidad política se muestra como un fraude y un engaño (Taylor, 1997: 364-365).

Así pues, el modo de hacer política que surge naturalmente de estas formas de exclusión -esté sustentada en la realidad o proyectada filosóficamente, aunque por lo general sea una mezcla de ambas-, evita la formación de compromisos en torno a alguna concepción del bien general.

Según Taylor (1996: 17-123), la única forma de dotar de un nuevo sentido a nuestra cultura y superar aquella desazón que la aqueja es rearticular la idea de la identidad moderna, algo que sólo podemos hacer seriamente si sobrepasamos la mera noción de lo que es ser un agente humano, una persona o un yo, para ahondar en la comprensión de cómo se han desarrollado las ideas del bien a las que esa noción se vincula.

Entendida de ese modo, la empresa que el filósofo canadiense acomete es, en buena medida, una tarea que pretende recuperar las descripciones morales en las cuales se sustentan nuestras creencias e intuiciones morales compartidas. Con todo, esa tarea no resulta nada sencilla debido a la estrechez de la filosofía moral contemporánea, que "ha tendido a centrarse en lo que es 
correcto hacer en vez de en lo que es bueno ser, en definir el contenido de la obligación en vez de la naturaleza de la vida buena; y no deja un margen conceptual para la noción del bien" (Taylor, 1996: 17).

Los seres humanos nos encontramos orientados hacia determinados fines y bienes, los cuales emergen de las intuiciones y creencias morales que dan contenido a las dimensiones fundamentales del pensamiento moral, las cuales, a su vez, se sustentan en una valoración fuerte que encuentra su explicación en una específica concepción ontológica del ser humano que arranca de algún marco referencial. Si todo esto es cierto, Taylor nos propone que el estudio de la ética debe encaminarse a una sistematización de la idea de identidad del yo y cómo se relaciona con una determinada noción del bien. Esta forma de proceder supone, desde luego, negar el subjetivismo moral.

Ahora bien, esa sistematización del concepto de la identidad moderna pasa necesariamente por la recuperación del ideal de la autenticidad (Taylor, 1994: 61-65; 1997: 294-299; 309-316). Taylor señala que las fuentes de ésta se encuentran en la noción del siglo XVIII de que las personas estamos dotadas de sentido moral, esto es, poseemos una capacidad de comprender el bien y el mal no como una cuestión de cálculo, sino como algo arraigado en nuestra propia naturaleza.

De este modo, la autenticidad exige dos rasgos inherentes: no puede florecer sin firmes lazos con los demás, pero al mismo tiempo excluye la validez de demandas que se encuentren más allá de las aspiraciones humanas, las cuales son contraproducentes para el ideal pues destruyen las condiciones indispensables para la realización de la autenticidad misma. Lo que Taylor quiere decir es que la definición de la identidad supone encontrar lo que resulta significativo para cada uno y nos diferencia de los demás, pero esta singularidad no descansa en el valor de la pura elección que hagamos de esos aspectos distintivos, sino contrastados con nuestros marcos de referencia ineludibles (Taylor, 1994: 72).

A menos que supongamos que algunas opciones poseen más valor que otras, la idea misma de autoelección cae en la trivialidad y, por ende, en la inconsistencia. El ideal de la autoelección sólo tiene sentido porque algunas cuestiones son más significativas que otras. No podría defender seriamente que nos elegimos a nosotros mismos porque preferimos escoger un filete con ensalada en vez de un guiso para cenar.

Y lo significativo no es algo que cada uno establezca, pues si fuera así nada sería significativo; por lo tanto, el ideal mismo de la autoelección como noción moral sería imposible. Se halla en la naturaleza más profunda de la propia modernidad su éxito y su miseria; inevitablemente el incremento de la libertad puede hacer que la gente se eleve o se degrade. 
La pregunta no es sólo si se puede defender la supervivencia de la identidad de un crisol de comunidades diferentes en contextos multiculturales, sino, además, si es posible exigir que todos reconozcamos igualitariamente el valor de cada una de ellas. Esta cuestión entonces debe llevarnos a preguntar por el valor que recibe o se le otorga a estas comunidades, valor en virtud del cual dichas comunidades podrían demandar el reconocimiento de su identidad.

Para llevar a cabo adecuadamente dichas evaluaciones del valor de una cultura, Taylor (1997: 199-220) habla de una "fusión de horizontes": debemos permitir que nuestros propios horizontes de sentido se amplíen en función de aquellos imbricados en la cultura a la cual nos aproximemos. De esta manera, aquello que en un principio se consideraba ya establecido desde nuestra propia perspectiva cultural tendrá, forzosamente, que verse modificado para intentar comprender el punto de vista del otro, desde su peculiar horizonte de sentido.

Desde el punto de vista de Taylor, la exigencia de reconocimiento se vuelve apremiante debido a los supuestos nexos entre el reconocimiento y la identidad, donde este último término designa algo equivalente a la interpretación que hace una persona de quién es y de sus características definitorias fundamentales como ser humano. La tesis de Taylor (1993: 30) es que nuestra identidad se moldea, en parte, por el reconocimiento o por la falta de éste que se constituye en una forma de opresión.

Al plantear un modelo narrativo para conceptualizar la identidad personal, su objetivo es corregir la abstracción del universalismo moral anclándolo en el contexto concreto de la comunidad y desarrollar un reconocimiento de las diferencias específicas de cada persona o grupo (Nájera-Pérez, 1999).

Ahora bien, el enfoque de la interseccionalidad también nos permite revisar los postulados de Taylor. En la medida que el autor sitúa el reconocimiento de las diferencias culturales como constitutivas de la identidad individual, desde la interseccionalidad, podemos plantear que él mismo ignora la construcción múltiple de la identidad dentro de distintas culturas y como tal no asume con coherencia un universalismo interactivo capaz de pensar al otro concreto, es decir, capaz de reconocer la pluralidad de modos de ser humano y de distinguir entre ellos, sin inhabilitar la validez política y moral de todas esas pluralidades y diferencias (Benhabib, 1990).

En este sentido, si asumimos que la identidad no sólo proviene de una cierta adscripción comunitaria, sino se entrecruza con otras fuentes de significado y ejes de diferenciación sobre los cuales se (re)producen las desigualdades, resulta pertinente criticar a Taylor el aspecto somático de cómo entiende la identidad. 
Benhabib (1990) nos entrega algunos elementos claves en esta dirección, al señalar que el filósofo, en su concepción de la identidad como narración, pone escasa atención al reconocimiento de una multiplicidad de factores (de género, de "raza"/etnia y de clase, etcétera) que determinan las experiencias y, de esta forma, la narración del "yo" para sí mismo y para los demás.

Es decir, en la medida que la fundación y conflictividad de las subjetividades está dominada por las categorías antes expuestas, cabe plantear la vigencia de la demanda del reconocimiento social de la identidad cultural propia de las personas, pueblos y culturas, expuestas por Taylor, en las complicidades socioculturales de definición o reconocimiento del otro/a en términos de género, etnicidad y diversidad cultural (Nash, 2001: 35).

De la misma forma, la máxima de Taylor de que todas las culturas son valiosas puede ser puesta en jaque desde una perspectiva interseccional, pues esto dependerá de quién hace la valoración, es decir, desde qué posición múltiple ocupe.

\section{Conclusiones}

El objetivo del artículo ha sido discutir un punto de vista alternativo desde donde analizar los límites y alcances de las teorizaciones en torno a la diversidad cultural - a partir del ejemplo de dos autores representativos del multiculturalismo liberal- y desde ahí proponer una alternativa crítica que sea un aporte al estudio sobre la diversidad cultural.

Al revisar los postulados de Kymlicka y Taylor ha sido posible identificar un excesivo énfasis en las diferencias culturales por sobre, y en desmedro de, otros ejes de diferenciación como el género y la clase social, por ejemplo. En este sentido, ambas posturas, representantes del multiculturalismo liberal, en su intento por confrontar la manera en que se responde a las necesidades y reivindicaciones de grupos diferenciados, corren el riesgo de desarrollar una política de reconocimiento que enfatice las diferencias culturales y, en última instancia, permita justificar situaciones de desigualdad.

De esta forma, aun cuando las ideas de homogeneización cultural pertenecen en su mayoría al pasado, queda pendiente la tarea de incentivar dentro del multiculturalismo el debate en relación con la construcción de lo cultural no como algo homogéneo con claras fronteras, y con una noción de identidad como proceso -y no como algo fijo y estable supuestamente anclado en contextos culturales específicos-.

El análisis llevado a cabo nos permite corroborar y concluir lo señalado por Nash (2001), respecto a que la explosión multicultural ha llevado a cierta simplificación del fenómeno y de los conceptos vinculados con el multicultu- 
ralismo; a la vez, ha ignorado a menudo un entendimiento del mismo como proceso de dinámicas sociales y culturales con un fuerte arraigo histórico y con dimensión de género.

En este sentido, se apuesta por una conceptualización del multiculturalismo que abogue por las afiliaciones múltiples de adscripción de una pluralidad de identidades, de culturas híbridas, complejas y en constante proceso de transformación, capaces de responder a las plurales experiencias e intersecciones de género, etnicidad y diversidad cultural en la sociedad de hoy.

También es necesario señalar que las posiciones liberales -además de aquellas nuevas formas de construcción identitaria que emergen desde la perspectiva posmoderna, entre las cuales encontramos, por ejemplo, las identidades híbridas- corren el riesgo de no cuestionar los contextos de desigualdad derivados del género, la clase o la "raza"/etnia en los que se sitúan las identidades minoritarias y mayoritarias.

La apuesta es reconocer que la inequidad no sólo se da entre culturas diferentes, sino también "dentro" de cada una de las culturas y, por lo tanto, es necesario distinguir los niveles de tratamiento de estas problemáticas. Por ejemplo, la diferencia étnica, como construcción social, se refiere a cosmovisiones distintas, lo cual implica estructuras y categorías para pensar y percibir lo real desde una determinada configuración. Dentro de cada cosmovisión, están presentes, marcadas por estas estructuras y categorías, las otras diferencias construidas socialmente, por lo cual su análisis y tratamiento debe tomar en cuenta este hecho.

En la tarea de proponer una alternativa crítica a la conceptualización de la diversidad cultural, creemos que el estudio y el tratamiento de ésta deben considerar el análisis de género como agente central de dichas experiencias. Parte importante de esta tarea es reflexionar sobre la compleja relación entre los objetivos de la perspectiva feminista contemporánea y las reivindicaciones de determinadas prácticas culturales que se inscriben en el debate respecto a la diversidad cultural y que pueden perjudicar a las mujeres.

Pero además se debe desafiar la representación del "hombre blanco europeo de clase alta" como norma y sujeto universal del pensamiento político y social occidental, a partir del cual se constituyó, en gran medida, el referente definitorio de los "otros" y se contribuyó a que esto se haga en términos de una relación jerarquizada respecto de cada grupo. Se trata de una perspectiva donde se mantenga el respeto con las culturas y con la igualdad y la diversidad de géneros.

Parte de esta perspectiva requiere poner atención en las representaciones culturales y prácticas sociales que delimitan la visión del otro. Por eso, dentro del análisis de la diversidad cultural es necesario tener en cuenta la 
intersección de los distintos ejes de diferenciación que permiten hablar de un universo de diversidades, las cuales, a su vez, son consecuencia de distintas relaciones de poder que no pueden dejarse de lado.

Parte esencial aquí es, además, hacer una lectura sobre las diferencias culturales y las desigualdades derivadas de éstas desde su relación con determinados ejes de estratificación social que tienen que ver con el género, la "raza"/etnia, la clase social, la sexualidad y la nación en la medida que, siguiendo a Fraser (1997), es la única manera de diseñar una concepción de la democracia radical que inspire credibilidad.

Sólo al integrar y conectar el estudio de la "raza"/etnia, la clase social y el género a la naturaleza de la construcción de la conciencia, a la producción de conocimiento y a los modos de opresión, el estudio de la diversidad cultural puede incluir una visión social que se extiende más allá de los intereses particulares de determinados grupos sociales.

De esta forma, se aboga por una política democrática con énfasis en la naturaleza específica de las diferencias, pero dentro de los principios de la igualdad y la justicia, los cuales sólo pueden ser alcanzados por el conjunto de la sociedad mediante la creación de puentes de actuación desde ámbitos distintos y colectivos sociales amplios (Nash, 2001).

Quisiéramos finalizar con algunas recomendaciones conceptuales que sirven como guía para el estudio de la diversidad cultural y que sintetizan los aportes de este trabajo:

1. Es necesario repensar el fenómeno de la diversidad cultural desde una perspectiva de la interseccionalidad y mediante un enfoque procesal: una visión monolítica puede ser provocada y perpetuada por políticas, agentes y estructuras de poder hegemónicos.

2. El empleo de este marco teórico de la interseccionalidad permite lecturas contextualizadas de poder. Se parte de la base que los sistemas de opresión son múltiples y simultáneos (patriarcado, capitalismo del consumo, supremacía racial, supremacía heterosexual), los cuales afectan tanto a las mujeres como a los hombres de manera diferenciada. Esto requiere ir más allá del mero establecimiento de la diversidad al buscar una diversidad que entienda la diferencia de poder cuando sea conceptualizada dentro del marco de un interés más alto por la justicia y el cambio social.

3. El concepto de interseccionalidad permite profundizar en aquellas discriminaciones y desigualdades que ocurren en grupos subordinados y oprimidos que se autodefinen como no hegemónicos. Sin embargo, y en la medida que pareciera ser que la diversidad cultural está siempre del lado de lo no hegemónico, el concepto de interseccionalidad nos hace notar la importancia de considerar también lo que ocurre dentro de los grupos hegemónicos. 
4. En análisis contextualizados sobre el estudio de la diversidad cultural sería de utilidad combinar el modelo interseccional con un análisis del tipo de poder que acompaña a las estructuras de opresión que impactan en, o incrementan, la discriminación hacia las minorías. Las formas de opresión sólo pueden ser comprendidas dentro de un contexto estructural, debido a que el modo como uno experimenta la raza, la clase social y el género dependen de sus intersecciones entre sí y con otras jerarquías de desigualdad. Esto permitiría enriquecer la reflexión teórica sobre tipos y prácticas de poder (ejercidos por instituciones y responsables políticos) y su vínculo con las múltiples y simultáneas formas de discriminación a las cuales se ven enfrentados.

5. Una ventaja significativa de este marco de análisis combinado (y análisis de poder) es que permite visibilizar de mejor manera la relación entre estructuras de desigualdad y los privilegios sociales resultantes de ellas. Por otra parte, provee nuevas luces sobre la posición de determinados individuos dentro de los grupos minoritarios.

6. Es importante seguir aclarando el marco conceptual y bagaje lingüístico con el que se aborda el fenómeno de la diversidad cultural. Los conceptos y terminologías tienen un valor político clave.

7. La ausencia de una perspectiva de género en el momento de teorizar puede redundar en la perpetuación de la diferenciación jerárquica entre los sexos y, por extensión, en otros tipos de divisiones identitarias. En términos prácticos, lo anterior puede operar en desmedro de la construcción de nuevos espacios de expresión para aquellas voces que tradicionalmente han sido silenciadas en la sociedad.

\section{Referencias}

Andersen, Margaret L. (2005), "Race, gender, and Class Stereotypes: New Perspectives on Ideology and Inequality”, en Revista Norteamérica, año 1, núm. 1, México: UNAM.

Anthias, Floya (1998), "Rethinking Social Divisions: Some Notes towards a Theoretical Framework”, en Sociological Review, vol. 46, núm. 3, EUA: Sage.

Anthias, Floya (2008), "Thinking through the lens of translocational positionality: an intersectionality frame for understanding identity and belonging", en Revista Translocations: Migration and Social Change, año 4, núm. 1, Ireland: DCU.

Anthias, Floya y Nira Yuval-Davis (1992), Racialized Boundaries: Race, Nation, Gender, Colour and Class and the Anti-Racist Struggle, London: Routledge.

Anzaldúa, Gloria (1987), Borderlands / La Frontera: The New Mestiza, San Francisco: Aunt Lute Books.

Banting, Keith y Will Kymlicka (2006), Multiculturalism and the Welfare State: Recognition and Redistribution in Contemporary Democracies, UK: Oxford University Press. 
Convergencia Revista de Ciencias Sociales, núm. 72, 2016, Universidad Autónoma del Estado de México

Barry, Brian (2002), Culture and Equality. An egalitarian Critique of Multiculturalism, Cambridge, Massachusetts, EUA: Harvard University Press.

Benería, Lourdes y Martha Roldán (1987), The crossroads of class \& gender: industrial homework, subcontracting, and household dynamics in Mexico City, Chicago: University of Chicago Press.

Benhabib, Seyla (1990), "El otro generalizado y el otro concreto: la controversia KolhbergGilligan y la teoría feminista”, en Benhabib, Seyla. y D. Cornell [eds.], Teoría Feminista y Teoria Crítica, Valencia: Alfons El Magnànim.

Benhabib, Seyla (2006), Las reivindicaciones de la cultura. Igualdad y diversidad en la era global, Buenos Aires y Madrid: Katz Barpal Editores.

Carens, Joseph H. (2000), Culture Citizenship, and Community. A Contextual Exploration of Justice as Evenhandedness, Oxford UK: Oxford University Press.

Crenshaw, Kimberlé (1994), "Mapping the Margins: Intersectionality, Identity Politics, and Violence Against Women of Color”, en M. Albertson Fineman y R. Mykitiuk [eds.], The Public Nature of Private Violence, New York: Routledge.

Curiel, Ochy (2008), “La crítica poscolonial desde las prácticas políticas del feminismo antirracista”, en Verschuur, C. [ed.], Vents d'Est, vents d'Ouest. Mouvements de femmes et féminismes anti-coloniaux, Ginebra: Graduate Institute of International and Development.

Curiel, Ochy (2014), “Género, raza, sexualidad: debates contemporáneos”. Colombia: Universidad del Rosario. Disponible en http://www.urosario.edu.co/urosario_ files/1f/1f1d1951-0f7e-43ff-819f-dd05e5fed03c.pdf [28 de enero de 2015].

Dávila-Figueroa, Ruth A. (2013), "Multiculturalismo y pueblos indígenas en zonas urbanas en Canadá: una reflexión sobre el reconocimiento y la redistribución”, en Norteamérica, vol. 8, núm. 2, México: UNAM.

Davis, Kathy (2008), "Intersectionality as buzzword: A sociology of science perspective on what makes a feminist theory successful”, en Feminist Theory, año 9, núm. 1, USA: Sage.

Delanty, Gerard (2010), Community, Londres: Routledge.

Deckha, Maneesha (2004), "Is Culture Taboo? Feminism, Intersectionality, and Culture Talk in Law”, en Canadian Journal of Women and the Law, vol. 16, núm. 1, Toronto: University of Toronto Press.

De Lucas, Javier (2001), “La(s) sociedad(es) multicultural(es) y los conflictos políticos y jurídicos", en La multiculturalidad, Madrid: Consejo General del Poder Judicial.

Fraser, Nancy (1997), Iustitia Interrupta. Reflexiones criticas desde la posición "postsocialista", Bogotá: Siglo del Hombre Editores.

Fraser, Nancy (2000), "Rethinking Recognition: Overcoming Displacement and Reification in Cultural Politics”, en New Left Reviews, núm. 3, Londres.

Femenías, María Luisa (2008), El género del multiculturalismo, Bernal: Universidad Nacional de Quilmes.

Guzmán-Ordaz, Raquel (2009), "Hacia un Análisis Interseccional de los Procesos Migratorios Feminizados y la Ciudadanía”, en Vásquez Bermúdez, I. [coord.], Investigación y Género. Avances en las Distintas Áreas del Conocimiento, España: Universidad de Sevilla.

Guzmán-Ordaz, Raquel (2011), El Paradigma Interseccional: Rutas Teórico Metodológicas para el Análisis de las Desigualdades Sociales, material inédito, en prensa. 
Sabina García-Peter y Luis Villavicencio-Miranda. Alcances y limites del multiculturalismo liberal desde un enfoque de género interseccional

Hancock, Ange Marie (2007), "Intersectionality as a normative and empirical paradigm", en Politics \& Gender, vol. 3, núm. 2, EUA: Cambridge Journals.

Kymlicka, Will (1989), "Liberal Individualism and Liberal Neutrality”, en Ethics, vol. 99, núm. 4, Chicago: University of Chicago Press.

Kymlicka, Will (1995), Multicultural Citizenship, Oxford: Oxford University Press.

Kymlicka, Will (1996), Ciudadanía multicultural. Una teoria liberal de los derechos de las minorias, España: Paidós.

Kymlicka, Will (2001), Politics in the Vernacular. Nationalism, Multiculturalism and Citizenship, UK: Oxford University Press.

Kymlicka, Will (2002), Contemporary Political Philosophy. An Introduction, Oxford: Oxford University Press.

Kymlicka, Will (2003), La politica vernácula. Nacionalismo, multiculturalismo y ciudadanía, Barcelona: Paidós.

Kymlicka, Will (2007), Multicultural Odysseys. Navigating the New International politics of Diversity, UK: Oxford University Press.

McCall, Leslie (2005), “The Complexity of Intersectionality”, en Signs: Journal of Women in Culture and Society, año 30, núm. 30, EUA: The University of Chicago Press.

Modood, Tariq (2007), Multiculturalism. A Civic Idea, UK: Polity Press.

Moller-Okin, Susan (1999), Is multiculturalism bad for women?, Princeton, New Jersey: Princeton Unuversity Press.

Moraga, Cherrie y Gloria Anzaldúa (1981), This bridge called my back, Bloomsbury, Londres: Persephone Press.

Nájera Pérez, E. (1999), “Concepciones y narrativas del yo", en Revista Themata, núm. 22, Sevilla: SPUS.

Nash, Marie (2001), "Diversidad, multiculturalismo e identidades. Perspectivas de género", en Nash, Marie y D. Marre [eds.], Multiculturalismos y género: perspectivas interdisciplinarias, Barcelona: Ediciones Bellaterra.

Nash, Jennifer C. (2008), "Re-thinking Intersectionality”, en Feminist Review, núm. 89, UK: Palgrave MacMillan.

Parekh, Bhikhu (1999), "Political Theory and the Multicultural Society", en Radical Philosophy, núm. 95, UK: Editorial Collective.

Parekh, Bhikhu (2002), "Barry and the Dangers of Liberalism”, en Kelly, P., Multiculturalism Reconsidered, Cambridge: Polity Press.

Parekh, Bhikhu (2006), Rethinking Multiculturalism. Cultural Diversity and Political Theory, UK: Palgrave MacMillan.

Parekh, Bhikhu (2008), A New Politics of Identity, Political Principles for an Independent World, London: Palgrave MacMillan.

Pérez, Federico (2007), "Will Kymlicka: la defensa del nacionalismo minoritario", en Astrolabio Revista Internacional de Filosofía, núm. 4, Barcelona: Universidad de Barcelona.

Pérez de la Fuente, Óscar (2005), Pluralismo culturaly derechos de las minorias, Madrid: Dykinson. Piastro, Julieta (2014), "Feminismo y género en el siglo XXI", en Revista de Psicología, vol. 32, núm.1, Barcelona: Facultat de Psicologia, Ciències de l'Educació i de l'Esport Blanquerna, Universitat Ramon Llull.

Raz, Joseph (2001), La ética en el ámbito de lo público, Barcelona: Gedisa. 
Rigat-Pflaum, María (2014), "Tres actos del feminismo. Nancy Fraser y los debates feministas de los últimos 40 años”, en Nueva Sociedad, núm. 252, Buenos Aires: Friedrich Ebert Stiftung.

Sánchez, Cristina (2002), "Feminismo y ciudadanía”, en Díaz, Elías y José Luis Colomer, Estado, justicia, derechos, Madrid: Alianza.

Shield, Stephanie A. (2008), "Gender: An intersectionality perspective, en Sex Roles, vol. 59, núm. 5-6, New York: Springer.

Song, Sarah (2007), Justice, Gender, and the Politics of Multiculturalism, Cambridge: Cambridge University Press.

Squella, Agustín et al. (2012), Curso de filosofía del derecho, Santiago: Editorial Jurídica de Chile.

Taylor, Charles (1983), Hegel y la sociedad moderna, México D.F.: Fondo de Cultura Económica.

Taylor, Charles (1992), Multiculturalism and the Politics of recognition: An Essay, Princeton: Princeton University Press.

Taylor, Charles (1993), El multiculturalismo y la politica del reconocimiento, México: Fondo de Cultura Económica.

Taylor, Charles (1994), La ética de la autenticidad, Barcelona: Paidós.

Taylor, Charles (1996), Fuentes del yo. La construcción de la identidad moderna, Barcelona: Paidós.

Taylor, Charles (1997), Argumentos filosóficos. Ensayo sobre el conocimiento, el lenguaje y la modernidad, Barcelona: Paidós.

Torbisco, Neus (2006), Group Rights as Human Rights. A Liberal Approach to Multiculturalism, Drodrecht: Springer.

Villavicencio, Luis (2009), "Algunas críticas a la idea de razón pública rawlsiana”, en Revista de Derecho, núm. 32, Valparaíso: Pontificia Universidad Católica de Valparaíso.

Villavicencio, Luis (2010a), "La relevancia moral de la identidad cultural", en Polis, vol. 9, núm. 26, Santiago: Universidad Bolivariana.

Villavicencio, Luis (2010b), "Privatizando la diferencia: El liberalismo igualitario y el pluralismo cultural", en Revista de Derecho, vol. 23, núm.1, Valdivia: Universidad Austral de Chile.

Villavicencio, Luis (2012), “Un diálogo intercultural más allá del multiculturalismo”, en Revista Opinión Jurídica, vol. 11, núm. 22, Medellín: Universidad de Medellín.

Villavicencio, Luis (2014), "Minorías étnico-culturales y derechos de las mujeres", en Revista de Ciencia Política, vol. 34, núm. 3, Santiago: Pontificia Universidad Católica de Chile, Instituto de Ciencia Política.

Winker, Gabriele y Nina Degele (2009), Intersektionalität: Zur Analyse sozialer Ungleichbeiten, Bielefeld: Transcript Verlag.

Young, Iris M. (1997), “A Multicultural Continuum: A Critique of Will Kymlicka's Ethnic Nation Dichotomy”, en Constellations, año 4, núm. 1, New York: The New School.

Young, Iris M. (2000), La justicia y la politica de la diferencia, Madrid: Cátedra.

Yuval-Davis, Nira (2006), "Intersectionality and Feminist Politics", en European Journal of Studies, vol. 13, núm. 3, EUA: Sage. 
Sabina García Peter. Doctora en Sociología. Institución de adscripción: Margherita-von-Brentano-Zentrum, Freie Universität Berlin, Alemania. Líneas de investigación: sociología de las ciencias sociales y humanidades, circulación de conocimientos, estudios de género e interseccionalidad, digitalización de la ciencia. Publicaciones recientes: Sabina García Peter, "La internacionalización del multiculturalismo liberal como estructura de posibilidad para su circulación en América Latina“, en Revista de Estudios Sociales, Bogotá (2016, en prensa); Sabina García Peter, "El multiculturalismo como modelo de gobernanza: Brokers, Estado y Academia", en Universitas Humanistica, vol. 82, núm. 82, Bogotá (2016); Jennifer Chan de Ávila, Sabina García Peter y Martha Zapata Galindo [eds.], Incluyendo sin excluir. Género y movilidad en la educación superior, Berlín: Tranvía (2013).

Luis Villavicencio Miranda. Doctor en Derecho. Institución de adscripción: Centro de Investigaciones de Filosofía del Derecho y Derecho Penal (CIFDEUV), Escuela de Derecho, Universidad de Valparaíso, Chile. Líneas de investigación: críticas comunitaristas, multiculturalistas y feministas al proyecto liberal de ciudadanía. Publicaciones recientes: "Minorías étnico-culturales y derechos de las mujeres", en Revista de Ciencia Política, vol. 34, núm. 3, Santiago: Pontificia Universidad Católica de Chile (2014); Las críticas comunitaristas al liberalismo igualitario. Un balance, Madrid: Dykinson-Universidad de Alcalá (2014); en coautoría con Alejandra Zúñiga Fajuri, "La violencia de género como opresión estructural", en Revista Chilena de Derecho, vol. 42, núm. 2, Santiago (2015).

Recepción: 27 de abril de 2015.

Aprobación: 25 de mayo de 2016. 
Convergencia Revista de Ciencias Sociales, núm. 72, 2016, Universidad Autónoma del Estado de México 